\title{
Clinical potential of mesenchymal stem/stromal cell-derived extracellular vesicles
}

\author{
Bernd Giebel $^{1}$, Lambros Kordelas ${ }^{2}$, Verena Börger ${ }^{1}$ \\ ${ }^{1}$ Institute for Transfusion Medicine, ${ }^{2}$ Department of Bone Marrow Transplantation, University Hospital Essen, University Duisburg-Essen, Essen, \\ Germany \\ Contributions: (I) Conception and design: All authors; (II) Administrative support: None; (III) Provision of study material or patients: None; (IV) \\ Collection and assembly of data: None; (V) Data analysis and interpretation: None; (VI) Manuscript writing: All authors; (VII) Final approval of \\ manuscript: All authors. \\ Correspondence to: PD Dr. rer. nat. Bernd Giebel. Institute for Transfusion Medicine, Universitätsklinikum Essen, Virchowstr. 179, 45147 Essen, \\ Germany. Email: bernd.giebel@uk-essen.de.
}

\begin{abstract}
Within the last two decades mesenchymal stem/stromal cells (MSCs) emerged after hematopoietic stem cells as the second most investigated and applied somatic stem cell entity so far. MSCs mediate immunosuppressive as well as pro-regenerative activities. Against the initial assumption, MSCs may not primarily exert their therapeutic functions in a cellular but rather in a paracrine manner. Here, extracellular vesicles $(\mathrm{EVs})$, such as exosomes and microvesicles, have been identified as major mediators of these paracrine effects. Meanwhile, MSC-EVs have been applied to an increasing amount of different animal models and were tested in a patient suffering from steroid-refractory acute graft-versus-host disease (acute GvHD) as well as in a patient cohort with chronic kidney disease. So far, the MSC-EV administration appears to be safe in humans and all tested animal models. Improvements were reported in all settings. Thus, MSC-EVs appear as promising novel therapeutic agents which might help to improve disease associated symptoms in millions of patients. Here, we review some of the milestones in the field, briefly discuss challenges and highlight clinical aspects of acute GvHD and its treatment with MSCs and MSC-EVs.
\end{abstract}

Keywords: Mesenchymal stem/stromal cells (MSC); cell therapy; extracellular vesicles (EVs); exosomes; microvesicles; immunomodulation; graft-versus-host disease (GvHD)

Received: 21 July 2017; Accepted: 27 August 2017; Published: 24 October 2017.

doi: $10.21037 /$ sci.2017.09.06

View this article at: http://dx.doi.org/10.21037/sci.2017.09.06

\section{Introduction}

Originally, stem cells are divided into two main categories, in embryonic stem cell (ES cells) and in somatic stem cells. ES cells are derivatives of blastocyst cells and are highly proliferative cells being programmed to create an organism with all its different embryonic tissues within a short period of time. ES cells are pluripotent and contain the potential to create each cell type of an organism $(1,2)$. In contrast, somatic stem cells are undifferentiated cells which are required for tissue homeostasis. Upon request somatic stem cells proliferate to create progenitor cells which differentiate into mature cell types to replace lost and damaged cells in affected tissues. Accordingly, somatic stem cells provide the fundament for the regenerative capabilities of somatic tissues and organs. In contrast to pluripotent cells, which nowadays can be created as induced pluripotent stem (iPS) cells by special reprogramming strategies $(3,4)$, somatic stem cells are restricted to create only selected cell types. To fulfil their function during the entire life span of an organism, an important feature of somatic stem cells is their ability to self-renew. In healthy tissues, the activation and self-renewal of endogenous somatic stem cells are highly controlled and biased. Any impairment which affects these processes might be fatal (5). Pathological increases in self-renewal and proliferation activities can result in 
tumor formation while a loss or massive reduction in selfrenewal and/or proliferation activities may lead to tissue degeneration and destruction.

During the last two decades, regenerative medicine has focused on strategies to treat degenerating tissues as well as injuries in tissues with limiting regenerative capabilities with exogenous stem cells. Initially, adapted to the principle of hematopoietic stem cell transplantation (6), the research in this area was focused on cell replacement strategies. It was considered that by substituting mature cell types being lost during the course of corresponding diseases or traumatic events, progeny of transplanted stem cells help to improve the clinical symptoms. At the turn of the century, when non-hematopoietic stem cell research became popular, experimental results implied that somatic stem cells contain much broader developmental potentials than they normally realize in the tissue they are harvested from. Papers entitled "Turning Brain into Blood" (7) and "Turning Blood into Brain" (8) implied that somatic stem cells contain the plasticity to create mature cell types of an array of different tissues $(9,10)$, and at least some MSClike subtypes contain pluripotent differentiation capabilities $(11,12)$. Since the usage of ES cells in regenerative medicine was hampered by their enormous teratogenic potential which initially was very challenging to be controlled (13), mesenchymal stem/stromal cells (MSCs) appeared as a promising cell source for cell replacement strategies. They did not reveal any teratogenic potential in animal models and can easily be raised from different human tissues, such as adult bone marrow and fat (14-17). Consequently, aside of hematopoietic stem cells, MSCs became the most investigated stem cell entity so far.

\section{MSCs and immunomodulation}

Due to the fact that acute injuries like ischemic stroke or myocardial infarction need to be treated very quickly and patient-derived off the shelf MSCs regularly do not exist, allogeneic MSC administration was considered as therapy of choice. Since allogeneic transplants in nonimmunocompromised recipients are regularly rejected by the recipients' immune system, a number of groups had started to investigate the interaction of different immune cell types with allogeneic MSCs. To this end, it was reported in 2002 that aside their multi-lineage differentiation capabilities MSCs contain immune modulating properties. MSCs were shown to inhibit the proliferation of stimulated $\mathrm{T}$ cells in a reversible manner (18-20). Cell-cell contact was not required for the suppression of $\mathrm{T}$ cell proliferation, implying that MSCs secrete T cell proliferation modulating factors (18). Following these initial studies, MSCs have been found to also regulate the activity of a number of different innate and adaptive immune cells. Apart of T cell proliferation they inhibit dendritic cell maturation and activation, recruit regulatory $\mathrm{T}$ cells and control $\mathrm{B}$ cell functions (21-24). Furthermore, MSCs were found to modulate the polarization of pro-inflammatory M1 to anti-inflammatory M2 macrophages and to inhibit NK cell activities (25-28). Coupled with results of many other studies, it became apparent that MSCs can effectively suppress pro-inflammatory immune responses and promote anti-inflammatory immune responses instead $(29,30)$. At the mechanistic level several cytokines, growth factors, enzymes and lipid mediators, such as interleukin-10 (IL-10), hepatocyte growth factor (HGF), transforming growth factor $\beta 1$ (TGF- $\beta 1$ ), tryptophan degrading enzyme indoleamine 2, 3-dioxygenase (IDO), the prostaglandin E2 (PGE2) and the human leukocyte antigen (HLA) class I molecule G (HLA-G), have been identified which are secreted by MSCs and promote anti-inflammatory immune responses $(18,20,27,28)$. Consequently, it was assumed that MSCs exert their immune modulatory functions by both, direct cellular contacts and the secretion of soluble factors $(29,30)$.

\section{MSCs in regenerative medicine and immunotherapy}

Coupled to the MSCs' multilineage potential and their immunomodulatory features the therapeutic impact of MSCs has been addressed in a huge collection of different animal models as well as in multiple clinical studies, including treatment of acute myocardial infarction, ischemic stroke, acute kidney failure and Crohn's disease as well as steroid-refractory acute Graft-versus-Host disease (acute GvHD), which arises as side effect of allogeneic hematopoietic stem cell transplantation therapies (Box 1) (37). Up to now, more than 800 clinical trials have been registered at the American National Institute of Health (NIH) intending to use MSCs for the treatment of a variety of different human diseases (clinicaltrials.gov).

The first study in which allogeneic MSCs had been transplanted into humans was reported in 1999; in the course of this study MSCs were administered to children with osteogenesis imperfecta (OI) (38). One year later a study was published, in which autologous MSCs had been applied to breast cancer patients after myeloablative therapy, simultaneously to autologous hematopoietic stem cell 
Box 1 Allogeneic hematopoietic stem cell transplantation and acute GvHD

\begin{abstract}
Allogeneic hematopoietic stem cell transplantation is a well-established therapeutic strategy for over 70 malignant and non-malignant hematologic diseases, including leukemia and lymphoma, which in many cases offers the only curative perspective (31). Regularly, allogeneic hematopoietic stem cell transplants (alloSCT) also contain immune cells, especially T cells, which on the one hand are required to kill residual malignant cells and avoid relapses; on the other hand, they can attack and destroy healthy tissues of the patient, such as the skin, the gastrointestinal tract and the liver. Thus, the immunologic effect of alloSCT can be characterized as a Janus face: the beneficial side, the graft-versus-leukemia (GvL) effect is often jeopardized by its reverse side: the graft-versus-host disease (GvHD). The incidence and severity of GvHD depend on a variety of factors. The most relevant risk factors are: the degree of donor-recipient human leukocyte antigen (HLA)-mismatches, donor and recipient age and sex as well as the stem cell source. Grade II-IV acute GvHD occur in approximately $35 \%$ of matched related alloSCT and in up to $50 \%$ of unrelated alloSCT (32)
\end{abstract}

\begin{abstract}
Despite numerous clinical studies, the standard immune suppressive regimens for prevention of acute GvHD have changed little in the last two decades. Standard prophylaxis typically involves improved HLA-matching of donor and recipient and consists of a short course of methotrexate (MTX) and cyclosporine (33). MTX is a folate antagonist with immunosuppressive potential and cyclosporine inhibits the enzyme calcineurin, which is important for the activation of T cells (34). If GvHD requires treatment, corticosteroids are the gold standard due to their strong anti-inflammatory and anti-lymphocyte properties. Corticosteroids are lympholytic and inhibit inflammatory cytokine cascades. Consequently, systemic administration of high-dose corticosteroids is associated with a high risk for morbidity due to various side-effects. Also the long-term treatment with lower doses can result in various complications such as hyperglycemia and diabetes mellitus, fluid retention and hypertension, muscle wasting, osteoporosis, psychosis, anxiety and depression. Complete remissions upon steroid therapy range from $25 \%$ to $50 \%$ and clearly correlate with overall survival (35)
\end{abstract}

Despite the fact that a plethora of new pharmacological substances have been introduced into clinical practice as second line therapy, steroid response is of utmost importance since the prognosis of steroid non-responder continues to be dismal with one-year survival around $30 \%$ (36). Given the poor prognosis of steroid-refractory acute GvHD there is still need for innovative therapy modalities

transplants (39). In 2004, LeBlanc and colleagues reported the usage of MSCs as immunomodulating therapeutic agent for the first time. The team infused allogeneic MSCs in a 9-year-old boy who developed a steroid-refractory acute GvHD after HLA-matched unrelated allogeneic hematopoietic stem cell transplantation. The acute GvHD was nonresponsive to corticosteroids and other immunosuppressive agents, however, infusion of MSCs from his HLA-haploidentical mother resulted in remarkable improvement of the acute GvHD symptoms (40). Although the patient was not cured and the acute GvHD recurred after some time, these symptoms were suppressed again following a second MSC infusion (40).

\section{MSCs in steroid-refractory acute GvHD}

Following the pioneering study of LeBlanc and colleagues several studies have investigated the impact of MSC administration in the treatment of steroid-resistant acute GvHD with controversial results (Box 2) (48). Some groups report beneficial effects, others did not observe any impact of the MSC therapy on acute GvHD patients at all. Notably, a phase III clinical trial (NCT00366145) failed to demonstrate efficacy of administered MSCs on acute GvHD symptoms (49). The different impacts of MSC therapies on acute GvHD symptoms might be attributed to qualitative differences in the applied MSCs. For now, MSC production and quality assurance as well as treatment protocols have not been standardized and the study design varied significantly between the different groups (47). Therefore, it is tempting to speculate that depending on the protocols and the tissue sources used, obtained MSCs differ in their therapeutic activities. According to our understanding, efforts are required to systematically compare impacts of culture conditions and application regiments on the therapeutic efficacy of obtained MSCs in the future.

\section{MSC in regenerative medicine}

Beneficial effects of transplanted MSCs have been observed in several studies in which MSCs were applied in regenerative settings $(37,50)$. Interestingly, similar as discussed for the immunomodulatory activities described above, it appears that, in contrast to the original cell replacement hypothesis, MSCs promote their proregenerative capabilities in a paracrine rather than a cellular manner $(50,51)$. Focusing on results obtained in acute myocardial infarction models, we briefly summarize some of the key-findings.

A number of studies, which were designed as cell replacement strategies, reported positive therapeutic impacts of administered MSCs in myocardial infarction 
Box 2 Effects of MSCs on acute GvHD symptoms in different studies

Dotoli and colleagues published the results of 46 patients treated with MSC infusion as salvage therapy for steroid-refractory acute GvHD III/IV (78\% grade IV), recently (41). Patients in this study received a median cumulative dose of $6.81 \times 10^{6} \mathrm{MSCs}$ per kg bodyweight in a median of three infusions. Overall, the authors report $13 \%$ complete remissions and $61 \%$ partial responses with no severe or late side effects which could be attributed to the administration of MSCs

Sánchez-Guijo and colleagues reported on the safety and efficacy of the administration of four sequential doses of cryopreserved bone marrow-derived MSCs from third-party donors as a second-line treatment for steroid-refractory acute GvHD (42). The MSC administration was well tolerated with the exception of a cardiac ischemic event that occurred twice in a patient with a prior history of cardiac ischemia. In terms of response seventeen patients (71\%) responded (11 complete and 6 partial responses), with a median time to response of 28 days after the first MSC dose, whereas seven patients did not respond

A phase I study comprised 15 pediatric and 25 adult patients with steroid-resistant grade II to IV GvHD treated with third party bone marrow-derived MSC (43). Patients received a median of three MSC infusions with a median cell dose of $1.5 \times 10^{6} / \mathrm{kg} \mathrm{per} \mathrm{infusion.} \mathrm{No} \mathrm{acute}$ toxicity was reported. Overall response rate at 28 days after the last MSC administration was $67.5 \%$, with $27.5 \%$ complete response

Zhao and colleagues observed a positive influence of bone marrow third-party donor MSCs on refractory acute GvHD and analyzed the immunomodulation effects in detail (44). They compared 28 patients with refractory acute GvHD who received MSC and 19 patients without MSC treatment and reported an overall response rate of $75 \%$ in the MSC group compared with $42.1 \%$ in the non-MSC group $(P=0.023)$. In addition to response regarding acute GvHD, the incidence and severity of chronic GvHD in the MSC group were lower than those in the non-MSC group ( $\mathrm{P}=0.045$ and $\mathrm{P}=0.005)$. The immunomodulation effects could be documented by higher regulatory $T$ cells frequencies in the MSC-treated patients than in the non-MSC group at 8 and 12 weeks after treatment

The outcome of other MSC application trials in acute GvHD have been summarized in several review articles. Chen and colleagues summarized the results of 13 studies with a total of 301 pediatric and adult patients and report an overall response rate (complete and partial remissions) after MSC treatment for steroid-refractory acute GvHD in 205 patients (66\%) (45). According to these review patients with skin steroid refractory acute GvHD showed a better response than patients with gastrointestinal $(P<0.05)$ or hepatic $(P<0.05)$ steroidrefractory acute GvHD. Patients with lower acute GvHD grade responded better (grade II vs. grade III-IV, $\mathrm{P}<0.05$ ). Moreover, the authors report a trend towards a better clinical response in children compared with adults

Munneke and colleagues have selected nine out of $255 \mathrm{MSC}$ studies according to specific quality criteria in their review. Of note, there is a significant variance in response rates from $8 \%$ to $83 \%$ (46). No serious side effects of MSC therapy were reported. Interestingly, complete response but not partial response to MSCs was associated with overall survival. Given the huge variance in response rates the need for prospective randomized trials is obvious

Rizk and co-workers attribute the uncertainty about and variance regrading MSC efficacy to a significant heterogeneity in MSC studies: age and diagnoses of patients, intensity and specifics of the conditioning regimens, degree of HLA matching and source of MSCs were not consistent (47). In addition, culture conditions and media supplements were highly variable and the characterization of MSCs was not uniform. Finally, treatment response criteria were not standardized either. Hence they concluded, it is crucial to develop and adhere to standardized criteria for MSC therapy in refractory GvHD

models $(52,53)$. Upon studying the bio-distribution of administered MSCs, it turned out that most intravenously and intra-arterial administered MSCs get rapidly trapped in the lung and are rarely recovered in other tissues (54-56). Thus, it was considered that comparable as in acute GvHD paracrine rather than cellular effects mediate the improvement of cardiac functions following myocardial infarction. Indeed, different labs recovered cardioprotective activities in MSC conditioned media (57-59).

Upon searching for the cardioprotective factors secreted by MSCs, Lee and colleagues identified the anti-inflammatory protein TNF- $\alpha$-induced protein 6 (TSG-6). Its siRNA mediated downregulation in MSCs abrogated the MSCs' cardioprotective effect while application of recombinant
TSG-6 could mick parts of the MSCs' therapeutic effect (54). In another approach to identify the cardioprotective components, the groups of Kleijn and Lim fractioned the supernatants of MSCs by filtration and recovered the cardioprotective activities in a fraction containing media-components larger than $1,000 \mathrm{kDa}$ with predicted diameters of 50 to $100 \mathrm{~nm}$. Upon analyzing the conditioned media by electron microscopy, the authors indeed identified vesicular structures of the expected size range, purified them by size exclusion technologies and confirmed their cardioprotective activities. At the molecular level the presence of exosomal marker molecules was confirmed. Accordingly, the authors concluded that the MSCs' cardioprotective activities reside 
in the identified exosome fraction (60). This was the second report which associated the MSCs' therapeutic activities with vesicles harvested from MSC conditioned media. The first report that MSCs act via vesicles was from the Camussi group. After showing that MSC conditioned media contain activities which improve the recovery from acute kidney failure, they have processed the conditioned media by ultracentrifugation and recovered the therapeutic activity within resulting ultracentrifugation pellets. Upon characterizing these fractions by electron microscopy, the authors found this fraction to be highly enriched for vesicles in the size range between $80 \mathrm{~nm}$ and $1 \mu \mathrm{m}$. At this time the authors termed these vesicles microvesicles (61).

\section{Extracellular vesicles (EVs)}

Cells can release a number of different vesicle types into their extracellular environment (62). Collectively they are named EVs $(63,64)$. Exosomes are small membrane vesicles $(70-150 \mathrm{~nm})$ that were discovered in 1983 as small vesicles that correspond to intraluminal vesicles (ILVs) of late endosomes, named multivesicular bodies (MVBs) or multivesicular endosomes. Upon studying transferrin trafficking it was observed that against the initial hypothesis a proportion of MVBs do not fuse with lysosomes to degrade their inner cargo but with the plasma membrane to release their cargo including the ILVs into the extracellular environment (65-67). Microvesicles derive as bud offs from the plasma membrane and have sizes between 100 and $1,000 \mathrm{~nm}$ (64). Other very prominent EVs are apoptotic bodies, vesicles which are formed when apoptotic cells are fragmented. They have said sizes of $500 \mathrm{~nm}$ to several micrometers (64), but according to unpublished data can also be as small as exosomes. EVs are found in all body liquids (68). Containing lipids, proteins and RNA, a proportion of EVs mediate targeted intercellular signaling in physiological and pathophysiological communication processes $(68,69)$. Other EVs might be formed as excretion vesicles allowing cells to excrete non-processable material (70). Also DNA containing EVs have been identified (71), which to our understanding might derive from apoptotic cells. The first experimental evidence that EVs act as signaling mediators in immune biological processes was provided by Raposo and colleagues in 1996 (72). Since then, EVs were found to mediate the interaction between various immune cell types and also between tumor and immune cells $(68,73)$. Depending on the cell source, EVs can promote or suppress pro-inflammatory responses $(68,69)$.

\section{MSC-EVs and their translation into the clinics}

\section{GvHD}

Getting aware of the two landmark papers highlighting the therapeutic potentials of MSC-EVs for the first time $(60,61)$ and knowing that EVs can exert immunomodulating functions (69), we wondered whether MSC-EVs also exert immunosuppressive functions of MSCs. To investigate this, we initially qualified the nanoparticle tracking analysis (NTA) as EV-quantification method and optimized a polyethylene glycol (PEG)-based large scale EV-preparation method $(74,75)$. Testing PEG purified MSC-EVs in a mixed lymphocyte reaction assay we observed that in the presence of MSC-EVs less immune cells secreted proinflammatory cytokines than in their absence. Thus, the in vitro data confirmed immunomodulatory properties of MSC-EVs (74). At this time, in the clinic, we were trying to suppress the symptoms of a steroid-refractory acute GvHD patient with different treatment options (Box 3). Since none of the conventional second line treatment options applied were effective, we considered the application of MSCs, but had to learn that the MSCs, which were commercially available at this time, failed to show efficiency in a phase III clinical study (49). Due to the disastrous condition of the patient and the lack of any other conventional treatment option, we decided to perform an individual treatment attempt with the MSC-EVs, whose immunomodulating abilities were confirmed in the mixed lymphocyte reaction assay (Box 3). As we had to consider that MSC-EV administration may cause unexpected side effects, MSC-EVs were administered in escalating doses. As reported in our manuscript, we did not observe any side effect, but the acute GvHD symptoms declined during the two weeks lasting treatment period significantly, the daily steroid dosage could be reduced from 125 to $30 \mathrm{mg}$ and the patient was stable for five months before some initial gut acute GvHD symptoms reappeared (Box 3) (74).

\section{Chronic kidney disease (CDK)}

A single centre study, randomized, placebo-controlled, phase II/III clinical pilot study, was performed with 40 patients in Cairo, Egypt (77). All patients, median age $24.65 \pm 0.75$ years (range, $19-34$ years), suffered from CDK for at least 6 months. None of the patients adopted chronic or recurrent infections during the last 12 months before treatment and had normal liver functions. The patients were divided into two groups, 20 patients each. The one 
Box 3 The first clinical application of MSC-EVs for steroid-refractory acute GvHD (74)

The 22-year old female patient, who was treated in 2011 with MSC-EVs, had received a first allogeneic stem cell transplantation (alloSCT) in February 2003 at the age of 13 due to a myelodysplastic syndrome (MDS RAEB-T). She obtained peripheral blood stem cells (PBSC) from an HLA-identical female donor following "conditioning" (the chemotherapeutical treatment required prior to alloSCT) with busulfan (alkylating agent), cyclophosphamide (chemotherapy with immunosuppressive properties) and melphalan (alkylating agent). After the first alloSCT she recovered hematologically with full donor chimerism and developed only limited mucosal GvHD. In October 2010 she suffered a relapse of the hematological disease as a secondary acute myeloid leukemia (AML) with $57 \%$ blasts in the bone marrow. FLT3- and NPM-mutations were detected. Consequently, in January 2011 a second alloSCT with PBSC from an HLA-identical male donor following conditioning with hyper-fractionated total body irradiation with 12 Gy and cyclophosphamide was performed. GvHD prophylaxis was standard cyclosporine (CSP, Calcineurin-inhibitor, the central prophylaxis against GvHD) and a short course of Methotrexate (MTX, inhibits T cell activation)

Following the second alloSCT, the patient developed a hyperacute GvHD of the skin grade IV with general erythema and bullae, grade IV, which initially responded sufficiently to high-dose steroids. Thus, the patient could be discharged with a dual immunosuppression of CSP and steroids. Unfortunately, the GvHD worsened shortly after discharge and the patient had to be admitted to hospital again in March 2011 with exacerbated skin GvHD and now also severe gastrointestinal involvement. She had therapy-refractory nausea and vomiting, abdominal cramping and diarrhea volumes reached up to five liters per day. The intestinal GvHD was repeatedly documented by endoscopy and histological examination

The severe GvHD did not respond to a number of immunosuppressive therapies. The patient immediately upon re-admission received high-dose steroids [up to $5 \mathrm{mg} / \mathrm{kg}$ body weight (BW)] with no obvious improvement. Therefore, a course of anti-thymocyte globulin (10 mg/kg BW) was applied over five days which also did not lead to a lasting response. Then, mycophenolate mofetil (MMF, inhibits the proliferation of T and B cells) and subsequently tacrolimus (inhibits production of IL-2 and the proliferation of T cells) were added. Since in some colon biopsies HHV-6, adeno and EBV virus DNA was detected, a therapy course with cidofovir (antiviral drug) was initiated in late April 2011, too. Unfortunately, all these measures did not lead to a relief in GvHD symptoms. Therefore, a course of infliximab, a chimeric monoclonal antibody that works against tumor necrosis factor alpha (TNF- $\alpha)$, (10 mg/kg BW once per week over a four-week period) was initiated. Additionally, 17 sessions of extra-corporeal photopheresis were performed over four months. This form of combined apheresis and photodynamic therapy is considered to damage T cells before they are reinfused into the patient, thus reducing the alloreactivity of respective T cells (76). Throughout this time the basic immunosuppression with steroids, mostly in combination with either tacrolimus or MMF, was continued. A therapy attempt with sirolimus, which as an mTOR inhibitor provides immunosuppressive properties, had to be discontinued due to increasing renal insufficiency

Given the history of this refractory and severe acute GvHD and the continuous suffering of the patient, we discussed in depth with the patient and her parents the possibility of an experimental approach to treat the steroid-refractory acute GvHD. A concept for an individual treatment attempt with MSC-EVs was developed and approved by the Legal Department of the University Hospital of Essen, Germany. We explained to the patient the current status of research regarding MSCs in general and the application via EVs in particular and emphasized the fact that there is no experience regarding this particular treatment modality at all. The patient agreed to the proposed treatment and gave her written consent. The patient received pre-medication with steroids and anti-histamines prior to each MSC-EV application and was closely monitored on our intensive care unit. MSC-EVs were applied in escalating doses, in total 7, over a period of two weeks

For the monitoring blood samples were taken before each MSC-EV application. Peripheral blood mononuclear cells (PBMCs) were isolated from these samples and transferred into a mixed leukocyte reaction (MLR) assay. Here, the cytokine responses of the different PBMC fractions, i.e., their release of IL-1 $\beta$, TNF- $\alpha$ and IFN- $\gamma$ towards K562 cells and their HLA-E*01:03 and HLA-B27 expressing counterparts, were analyzed simultaneously. Remarkably, following the third MSC-EV application the patient's PBMCs cytokine response towards the different K562 variants was clearly reduced. Compared to the cytokine responses of the patient's PBMCs before the MSC-EV therapy, the numbers of IL-1 $\beta$, TNF- $\alpha$ and IFN- $\gamma$ producing PBMCs were reduced more than fifty percent after the last application $(\mathrm{P}<0.0001)$

To monitor the effects of the MSC-EV therapy on the cytokine release in the peripheral blood, the blood plasma samples were analyzed for their content of pro- and anti-inflammatory cytokines or immune modulatory proteins, respectively. Remarkably, during the course of the therapy a clear reduction of the pro-inflammatory cytokines IL-6, IL-8 and IL-17A was recorded. In line with the reduced anti-inflammatory cytokine reaction during the course of MSC-EV therapy, the clinical GVHD symptoms improved significantly shortly after the start of the MSC-EV therapy: the skin manifestation clearly lost activity, nausea, vomiting and diarrhea were dramatically reduced. The cutaneous and mucosal GvHD showed a remarkable response within two weeks, which was stable even after 16 weeks following the MSC-EV therapy. The diarrhea volume, too, was objectively reduced after the MSC-EV therapy. Due to the clinical response the dosage of the steroids could be reduced from $125 \mathrm{mg} / \mathrm{d}$ before to $30 \mathrm{mg} / \mathrm{d}$ after the therapy. We have to admit that we did not cure the patient long-term. Most likely, due to the extensive immune deficiency five months later the patient acquired an encephalitis and pneumonia and died with multiorgan failure 
group received treatment with two doses of MSC-EVs (treatment interval: one week), one intravenously and one intra-arterial. The other group, the control group, received one saline injection intravenously. No MSC$\mathrm{EV}$ therapy related side effects were recorded during a one year follow up in any of the patients. One year after the therapy, CDK symptoms-measured by the estimated glomerular filtration rates (eGFR), the urinary albumin to creatinine ratio and the blood urea and serum creatinine levels-were improved in a non-significant manner in the MSC-EV treated but not in the control group. Taking immunomodulatory effects of the MSC-EV therapy into account, the authors analysed TGF- $\beta$, IL-10 and TNF- $\alpha$ levels 12 weeks and one year after the MSC$\mathrm{EV}$ treatment. Compared to the plasma levels in patients before treatment, the anti-inflammatory cytokines TGF- $\beta$ and IL-10 levels were significantly increased and those of the pro-inflammatory cytokine TNF- $\alpha$ significantly decreased in patients of the MSC-EV treated but not in those of the control group. Of note, in the MSC-EV treated group TGF- $\beta$ and IL-10 levels were higher at 12 weeks following MSC-EV treatment than after one year. In contrast, TNF- $\alpha$ levels were slightly lower after one year than after 12 weeks. Biopsy samples of 3 MSC-EV treated patients were analyzed and compared to biopsies of patients of the control group. In contrast to the biopsies of the control group patients, the biopsies of the MSC-EV treated patients revealed the presence of activated renal progenitor cells. Thus, a co-incidence of anti-inflammatory and proregenerative activities was observed, which coincides with the improvement of the disease symptoms.

It is worth mentioning that the clinical symptoms have been improved in some patients immediately following the first MSC-EV administration and in others in a delayed manner following the second MSC-EV application. Since only little information about the nature of the MSC-EV preparations is given, which unfortunately does not comply with the quality assurance recommendations of the International Society of Extracellular Vesicles (ISEV) for EV preparations (78), the differences might either depend on the quality of MSC-EV samples and/or inter-individual differences of the patients including variations in the course of their diseases. Apart of our GvHD treatment attempt and the CDK study, we are not aware about any study in which stem cell-derived EVs were administered to humans. A phase II-III clinical study entitled "Effect of Microvesicles and Exosomes Therapy on $\beta$-cell Mass in Type I Diabetes Mellitus (T1DM)" has been registered in 2014 at the NIH
(NCT02138331) by the same group which has performed the CDK study. However, results of this study have apparently not been published, yet.

\section{Safety of MSC-EV administration}

In total, we are aware of 41 patients which have been treated with MSC-EVs $(74,77)$, none of which showed any side effect. In addition, we and others have applied MSC-EVs to an increasing amount of different animal models for various diseases. We did not detect any side effects of the MSCEV administration in any of our animal models (79-81), even though MSC-EVs prepared with the PEG method were applied in up to 100 fold higher doses per Gramm body weight to the animals than to the respective acute GvHD patient. Furthermore, irrespectively of the protocol used for the MSC-EV purification, we are not aware of any reported side effects of the MSC-EV administration in any of the published animal models. Thus, we consider the MSC-EV administration as principally safe (82).

\section{Stem cell versus stem cell-EV therapy}

As described before, MSCs have been frequently applied in clinical trials. Apparently, a huge portion of their therapeutic activities is mediated by their EVs. Indeed, evidence has been provided that MSC-EVs exert immunomodulatory as well as pro-regenerative effects and efficiently mimic therapeutic effects of MSCs (82). Due to the huge amount of clinical trials using MSCs to treat various diseases in humans, there is much more experience of how MSCs can be translated into the clinics than for EVs. Furthermore, regulatory requirements for the clinical grade production of MSCs have been met. MSCs intended to be used as therapeutic agent are classified as advanced therapy medicinal product (ATMPs) by legislation $(83,84)$. Connected to this, tight regulations have been released by official regulatory instances such as European Medicines Agency (EMA) and the Food and Drug Administration (FDA), which need to be fulfilled for the standardized production and quality assurance of cellular therapeutics (85). One of several prerequisite for therapeutic agents to be defined as ATMPs is that they either contain a nucleus or an active transgenic component. Since MSC-EVs, at least from genetically non-engineered MSCs, neither contain a nucleus nor a transgenic product they do not fall into the currently defined ATMP category. Although, MSC-EVs might be 
considered as a sub-group of "biological medicines", they have not been categorized in an international accepted manner, yet. Currently, requirements for their production and application are regulated in country- or even countywise manners. Still, due to the novelty of the EV-field, we are not aware about any official guidelines for EV-based therapeutics not falling into the ATMP category. So far, recommendations for the production and application of EV-based therapeutics have just been provided by ISEV members and delegates of the EU COST action on "Microvesicles and Exosomes in Health and Disease (ME$\mathrm{HaD})$ " in a joined position paper and its follow up $(85,86)$.

So, the question might arise, why to translate MSCEVs into the clinics, if MSCs exert comparable therapeutic effects. To our understanding MSC-EVs provide several key advantages over cellular products with maybe comparable therapeutic activities, which justify efforts to translate EVs into the clinics:

* With average sizes below $200 \mathrm{~nm}$, EVs can be sterilized by filtration. This should massively reduce the risk of biological contamination of respective therapeutics. Accordingly, the regulatory requirements for the clinical grade production might be less restrictive for EVs than for ATMPs. Lower clean room standards than those for the ATMP production might be sufficient to expand EV-releasing stem cells and to purify released EVs from conditioned media. Maybe it will be sufficient to only perform the sterilization and the subsequent packing of EV-products under high clean room standards (85).

* In contrast to cellular products EVs cannot self-replicate and thus lack any endogenous tumor-formation potential (85). Although MSC administration appears to be safe, application of other somatic stem cell entities such as olfactory mucosal cells, which following transplantation in a spinal cord-injury patient formed a tumor at the injection side (87), underscores potential risks of somatic stem cell therapies. It has to be considered that most clinical MSC trials have been performed within the last ten years, so that long-term risks have not been evaluated, yet.

* Biological features and functions of cells can in principal be affected and re-programmed by environmental factors. Since EVs lack elaborated metabolic activities, it appears less likely that their function can be reprogrammed by the environment. Thus the biological activity and functional properties of EVs can be defined more precisely than for cells (85).

* EVs are much easier to handle than cells. Freezing, thawing and storage conditions appear to be less critical for the EVs than for cells (85). For the bed-side preparation of cellular transplants the personal has to be specifically trained which might be dispensable for the bed-side preparation of EVbased therapeutics.

* Therapeutic EVs might be produced from supernatants of cell lines, whose cells should not be used for cellular therapies themselves. Thus, EVs can much easier be produced in a scaled manner than cellular therapeutics (85).

\section{Summary and conclusions}

MSC-EVs contain immunomodulatory activities and promote regenerative processes, apparently in a comparable manner than MSCs. EV-based therapeutics provide a number of advantages over cellular therapeutics, but due to the novelty of the field internationally accepted guideless for their clinical grade production, quality assurance and application are lacking. Apart of the regulatory issues there are a number of challenges which are currently addressed in several laboratories intending to translate $\mathrm{EV}$-products into the clinics.

For example, classically, EVs are prepared from small sample sizes by centrifugation-based methods, especially by differential centrifugation (88). Due to limitations in rotor sizes and due to observations that ultracentrifugation might affect the integrity of EVs, ultracentrifugation-based methods might not be the methods of choice for the clinical grade production of EVs. Currently, several groups try to establish novel methods for the EV purification in closed systems such as tangential flow filtration $(89,90)$.

Another aspect in the production of EV-based therapeutics is the quality assurance. Although data from several animal models and the reported human studies suggest that MSC-EVs exert therapeutic effects without causing side effects, appropriate quality control criteria have to be set up, which ensure the quality, safety and efficacy of the generated EV-products.

As comprehensive discussions about the pending requirements that need to be fulfilled to translate $\mathrm{EVs}$ into the clinics just have been published recently by experts in the field, we like to refer to these publications and do 
not repeat these discussions here $(85,86)$. To our opinion, EVs and especially MSC-EVs provide a powerful novel therapeutic agent for many degenerative and inflammatory diseases, which might help to improve disease associated symptoms of millions of patients. It will be interesting to learn whether EVs from other somatic stem cell entities or from derivatives of pluripotent stem cells will also provide therapeutic functions and whether depending on the disease might exceed the effect of MSC-EVs.

\section{Acknowledgements}

For the collaboration and general support, we would like to thank our colleagues from our institutes, especially Prof. Peter Horn and Prof. Dietrich Beelen, as well as all collaboration partners. For the financial support of our EV research, we are grateful to the Medical Faculty of the University of Duisburg-Essen (IFORES), the Volkswagen Foundation, the Stem Cell Network North Rhine Westphalia and the LeitmarktAgentur.NRW and European Union (European Regional Development Fund 2014-2020), as well as to the European Union for the EU program ME$\mathrm{HaD}$ (COST Action BM1202).

\section{Footnote}

Conflicts of Interest: The authors have no conflict of interest to declare.

\section{References}

1. Thomson JA, Itskovitz-Eldor J, Shapiro SS, et al. Embryonic stem cell lines derived from human blastocysts. Science 1998;282:1145-7.

2. Reubinoff BE, Pera MF, Fong CY, et al. Embryonic stem cell lines from human blastocysts: somatic differentiation in vitro. Nat Biotechnol 2000;18:399-404.

3. Takahashi K, Yamanaka S. Induction of pluripotent stem cells from mouse embryonic and adult fibroblast cultures by defined factors. Cell 2006;126:663-76.

4. Takahashi K, Tanabe K, Ohnuki M, et al. Induction of pluripotent stem cells from adult human fibroblasts by defined factors. Cell 2007;131:861-72.

5. Görgens A, Giebel B. Self-Renewal of Primitive Hematopoietic Cells: A Focus on Asymmetric Cell Division. in Umbilical Cord Blood: A Future for Regenerative Medicine? World Scientific 2015.

6. Singh AK, McGuirk JP. Allogeneic Stem Cell
Transplantation: A Historical and Scientific Overview. Cancer Res 2016;76:6445-6451.

7. Bjornson CR, Rietze RL, Reynolds BA, et al. Turning brain into blood: a hematopoietic fate adopted by adult neural stem cells in vivo. Science 1999;283:534-7.

8. Mezey E, Chandross KJ, Harta G, et al. Turning blood into brain: cells bearing neuronal antigens generated in vivo from bone marrow. Science 2000;290:1779-82.

9. Lemischka I. Rethinking somatic stem cell plasticity. Nat Biotechnol 2002;20:425.

10. Wei G, Schubiger G, Harder F, et al. Stem cell plasticity in mammals and transdetermination in Drosophila: common themes? Stem Cells 2000;18:409-14.

11. Kögler G, Sensken S, Airey JA, et al. A new human somatic stem cell from placental cord blood with intrinsic pluripotent differentiation potential. J Exp Med 2004;200:123-35.

12. Jiang $Y$, Jahagirdar BN, Reinhardt RL, et al. Pluripotency of mesenchymal stem cells derived from adult marrow. Nature 2002;418:41-9.

13. Cunningham JJ, Ulbright TM, Pera MF, et al. Lessons from human teratomas to guide development of safe stem cell therapies. Nat Biotechnol 2012;30:849-57.

14. Pittenger MF, Mackay AM, Beck SC, et al. Multilineage potential of adult human mesenchymal stem cells. Science 1999;284:143-7.

15. Prockop DJ. Marrow stromal cells as stem cells for nonhematopoietic tissues. Science 1997;276:71-4.

16. Caplan AI, Bruder SP. Mesenchymal stem cells: building blocks for molecular medicine in the 21st century. Trends Mol Med 2001;7:259-64.

17. Porada CD, Zanjani ED, Almeida-Porad G. Adult mesenchymal stem cells: a pluripotent population with multiple applications. Curr Stem Cell Res Ther 2006;1:365-9.

18. Di Nicola M, Carlo-Stella C, Magni M, et al. Human bone marrow stromal cells suppress T-lymphocyte proliferation induced by cellular or nonspecific mitogenic stimuli. Blood 2002;99:3838-43.

19. Bartholomew A, Sturgeon C, Siatskas M, et al. Mesenchymal stem cells suppress lymphocyte proliferation in vitro and prolong skin graft survival in vivo. Exp Hematol 2002;30:42-8.

20. Meisel R, Zibert A, Laryea M, et al. Human bone marrow stromal cells inhibit allogeneic T-cell responses by indoleamine 2,3-dioxygenase-mediated tryptophan degradation. Blood 2004;103:4619-21.

21. Jiang XX, Zhang Y, Liu B, et al. Human mesenchymal 
stem cells inhibit differentiation and function of monocytederived dendritic cells. Blood 2005;105:4120-6.

22. Di Ianni M, Del Papa B, De Ioanni M, et al. Mesenchymal cells recruit and regulate T regulatory cells. Experimental hematology 2008;36:309-18.

23. Casiraghi F, Azzollini N, Cassis P, et al. Pretransplant infusion of mesenchymal stem cells prolongs the survival of a semiallogeneic heart transplant through the generation of regulatory T cells. J Immunol 2008;181:3933-46.

24. Corcione A, Benvenuto F, Ferretti E, et al. Human mesenchymal stem cells modulate B-cell functions. Blood 2006;107:367-72.

25. Németh K, Leelahavanichkul A, Yuen PS, et al. Bone marrow stromal cells attenuate sepsis via prostaglandin $\mathrm{E}(2)$-dependent reprogramming of host macrophages to increase their interleukin-10 production. Nat Med 2009;15:42-9.

26. Spaggiari GM, Capobianco A, Becchetti S, et al. Mesenchymal stem cell-natural killer cell interactions: evidence that activated NK cells are capable of killing MSCs, whereas MSCs can inhibit IL-2-induced NK-cell proliferation. Blood 2006;107:1484-90.

27. Selmani Z, Naji A, Zidi I, et al. Human leukocyte antigen-G5 secretion by human mesenchymal stem cells is required to suppress $\mathrm{T}$ lymphocyte and natural killer function and to induce CD4+CD25highFOXP3+ regulatory T cells. Stem Cells 2008;26:212-22.

28. Aggarwal S, Pittenger MF. Human mesenchymal stem cells modulate allogeneic immune cell responses. Blood 2005;105:1815-22.

29. Le Blanc K, Mougiakakos D. Multipotent mesenchymal stromal cells and the innate immune system. Nat Rev Immunol 2012;12:383-96.

30. Wang Y, Chen X, Cao W, et al. Plasticity of mesenchymal stem cells in immunomodulation: pathological and therapeutic implications. Nat Immunol 2014;15:1009-16.

31. Appelbaum FR. Haematopoietic cell transplantation as immunotherapy. Nature 2001;411:385-389.

32. Koreth J, Antin JH. Current and future approaches for control of graft-versus-host disease. Expert Rev Hematol 2008;1:111.

33. Yerushalmi R, Shem-Tov N, Danylesko I, et al. The combination of cyclosporine and mycophenolate mofetil is less effective than cyclosporine and methotrexate in the prevention of acute graft-versus host disease after stemcell transplantation from unrelated donors. Am J Hematol 2017;92:259-268.

34. Blazar BR, Murphy WJ, Abedi M. Advances in graft- versus-host disease biology and therapy. Nat Rev Immunol 2012;12:443-58.

35. Deeg HJ. How I treat refractory acute GVHD. Blood 2007;109:4119-26.

36. Bacigalupo A. Management of acute graft-versus-host disease. Br J Haematol 2007;137:87-98.

37. Singer NG, Caplan AI. Mesenchymal stem cells: mechanisms of inflammation. Annual review of pathology 2011;6:457-78.

38. Horwitz EM, Prockop DJ, Fitzpatrick LA, et al. Transplantability and therapeutic effects of bone marrowderived mesenchymal cells in children with osteogenesis imperfecta. Nat Med 1999;5:309-13.

39. Koç ON, Gerson SL, Cooper BW, et al. Rapid hematopoietic recovery after coinfusion of autologousblood stem cells and culture-expanded marrow mesenchymal stem cells in advanced breast cancer patients receiving high-dose chemotherapy. J Clin Oncol 2000;18:307-16.

40. Le Blanc K, Rasmusson I, Sundberg B, et al. Treatment of severe acute graft-versus-host disease with third party haploidentical mesenchymal stem cells. Lancet 2004;363:1439-41.

41. Dotoli GM, De Santis GC, Orellana MD, et al. Mesenchymal stromal cell infusion to treat steroidrefractory acute GvHD III/IV after hematopoietic stem cell transplantation. Bone Marrow Transplant 2017;52:859-862.

42. Sánchez-Guijo F, Caballero-Velázquez T, López-Villar $\mathrm{O}$, et al. Sequential third-party mesenchymal stromal cell therapy for refractory acute graft-versus-host disease. Biol Blood Marrow Transplant 2014;20:1580-5.

43. Introna $M$, Lucchini G, Dander E, et al. Treatment of graft versus host disease with mesenchymal stromal cells: a phase I study on 40 adult and pediatric patients. Biol Blood Marrow Transplant 2014;20:375-81.

44. Zhao K, Lou R, Huang F, et al. Immunomodulation effects of mesenchymal stromal cells on acute graft-versus-host disease after hematopoietic stem cell transplantation. Biol Blood Marrow Transplant 2015;21:97-104.

45. Chen X, Wang C, Yin J, et al. Efficacy of mesenchymal stem cell therapy for steroid-refractory acute graft-versushost disease following allogeneic hematopoietic stem cell transplantation: a systematic review and meta-analysis. PLoS One 2015;10:e0136991.

46. Munneke JM, Spruit MJ, Cornelissen AS, et al. The potential of mesenchymal stromal cells as treatment for severe steroid-refractory acute graft-versus-host 
disease: a critical review of the literature. Transplantation 2016;100:2309-2314.

47. Rizk M, Monaghan M, Shorr R, et al. Heterogeneity in studies of mesenchymal stromal cells to treat or prevent graft-versus-host disease: a scoping review of the evidence. Biol Blood Marrow Transplant 2016;22:1416-1423.

48. Baron F, Storb R. Mensenchymal Stromal Cells: A New Tool against Graft-versus-Host Disease? Biol Blood Marrow Transplant 2012;18:822-840.

49. Galipeau J. The mesenchymal stromal cells dilemma--does a negative phase III trial of random donor mesenchymal stromal cells in steroid-resistant graft-versus-host disease represent a death knell or a bump in the road? Cytotherapy 2013;15:2-8.

50. D'souza N, Rossignoli F, Golinelli G, et al. Mesenchymal stem/stromal cells as a delivery platform in cell and gene therapies. BMC Med 2015;13:186.

51. Caplan AI, Correa D. The MSC: an injury drugstore. Cell Stem Cell 2011;9:11-5.

52. Pittenger MF, Martin BJ. Mesenchymal stem cells and their potential as cardiac therapeutics. Circ Res 2004;95:9-20.

53. Tomita S, Li RK, Weisel RD, et al. Autologous transplantation of bone marrow cells improves damaged heart function. Circulation 1999;100:II247-56.

54. Lee RH, Pulin AA, Seo MJ, et al. Intravenous hMSCs improve myocardial infarction in mice because cells embolized in lung are activated to secrete the antiinflammatory protein TSG-6. Cell Stem Cell 2009;5:54-63.

55. Schrepfer S, Deuse T, Reichenspurner H, et al. Stem cell transplantation: the lung barrier. Transplant Proc 2007;39:573-6.

56. Gao J, Dennis JE, Muzic RF, et al. The dynamic in vivo distribution of bone marrow-derived mesenchymal stem cells after infusion. Cells Tissues Organs 2001;169:12-20.

57. Gnecchi M, He H, Noiseux N, et al. Evidence supporting paracrine hypothesis for Akt-modified mesenchymal stem cell-mediated cardiac protection and functional improvement. FASEB J 2006;20:661-669.

58. Gnecchi M, He H, Liang OD, et al. Paracrine action accounts for marked protection of ischemic heart by Aktmodified mesenchymal stem cells. Nat Med 2005;11:367-8.

59. Timmers L, Lim SK, Arslan F, et al. Reduction of myocardial infarct size by human mesenchymal stem cell conditioned medium. Stem cell research 2007;1:129-37.

60. Lai RC, Arslan F, Lee MM, et al. Exosome secreted by MSC reduces myocardial ischemia/reperfusion injury. Stem Cell Res 2010;4:214-22.

61. Bruno S, Grange C, Deregibus MC, et al. Mesenchymal stem cell-derived microvesicles protect against acute tubular injury. J Am Soc Nephrol 2009;20:1053-67.

62. Kim DK, Lee J, Kim SR, et al. EVpedia: a community web portal for extracellular vesicles research. Bioinformatics 2015;31:933-9.

63. Gould SJ, Raposo G. As we wait: coping with an imperfect nomenclature for extracellular vesicles. J Extracell Vesicles 2013;2.

64. Raposo G, Stoorvogel W. Extracellular vesicles: exosomes, microvesicles, and friends. J Cell Biol 2013;200:373-83.

65. Pan BT, Johnstone RM. Fate of the transferrin receptor during maturation of sheep reticulocytes in vitro: selective externalization of the receptor. Cell 1983;33:967-78.

66. Harding C, Heuser J, Stahl P. Receptor-mediated endocytosis of transferrin and recycling of the transferrin receptor in rat reticulocytes. J Cell Biol 1983;97:329-39.

67. Johnstone RM, Adam M, Hammond JR, et al. Vesicle formation during reticulocyte maturation. Association of plasma membrane activities with released vesicles (exosomes). J Biol Chem 1987;262:9412-20.

68. Yáñez-Mó M, Siljander PR, Andreu Z, et al. Biological properties of extracellular vesicles and their physiological functions. J Extracell Vesicles 2015;4:27066.

69. Ludwig AK, Giebel B. Exosomes: small vesicles participating in intercellular communication. Int J Biochem Cell Biol 2012;44:11-5.

70. Giebel B. On the function and heterogeneity of extracellular vesicles. Ann Transl Med 2017;5:150.

71. Thakur BK, Zhang H, Becker A, et al. Double-stranded DNA in exosomes: a novel biomarker in cancer detection. Cell Res 2014;24:766-9.

72. Raposo G, Nijman HW, Stoorvogel W, et al. B lymphocytes secrete antigen-presenting vesicles. J Exp Med 1996;183:1161-72.

73. Robbins PD, Morelli AE. Regulation of immune responses by extracellular vesicles. Nat Rev Immunol 2014;14:195-208.

74. Kordelas L, Rebmann V, Ludwig AK, et al. MSC-derived exosomes: a novel tool to treat therapy-refractory graftversus-host disease. Leukemia 2014;28:970-3.

75. Sokolova V, Ludwig AK, Hornung S, et al. Characterisation of exosomes derived from human cells by nanoparticle tracking analysis and scanning electron microscopy. Colloids Surf B Biointerfaces 2011;87:146-50.

76. Mohammadi S, Malek Mohammadi A, Norooznezhad $\mathrm{AH}$, et al. Extra corporeal photochemotherapy in steroid refractory graft versus host disease: A review of guidelines and recommendations. Transfus Apher Sci 2017;56:376-384.

77. Nassar W, El-Ansary M, Sabry D, et al. Umbilical cord 
mesenchymal stem cells derived extracellular vesicles can safely ameliorate the progression of chronic kidney diseases. Biomater Res 2016;20:21.

78. Lötvall J, Hill AF, Hochberg F, et al. Minimal experimental requirements for definition of extracellular vesicles and their functions: a position statement from the International Society for Extracellular Vesicles. J Extracell Vesicles 2014;3:26913.

79. Drommelschmidt K, Serdar M, Bendix I, et al. Mesenchymal stem cell-derived extracellular vesicles ameliorate inflammation-induced preterm brain injury. Brain Behav Immun 2017;60:220-232.

80. Ophelders DR, Wolfs TG, Jellema RK, et al. Mesenchymal stromal cell-derived extracellular vesicles protect the fetal brain after hypoxia-ischemia. Stem Cells Transl Med 2016;5:754-63.

81. Doeppner TR, Herz J, Gorgens A, et al. Extracellular vesicles improve post-stroke neuroregeneration and prevent postischemic immunosuppression. Stem Cells Transl Med 2015;4:1131-43.

82. Börger V, Bremer M, Ferrer-Tur R, et al. Mesenchymal stem/stromal cell-derived extracellular vesicles and their potential as novel immunomodulatory therapeutic agents. Int J Mol Sci 2017;18.pii:E1450.

83. Reflection paper on classification of advanced therapy

doi: $10.21037 /$ sci.2017.09.06

Cite this article as: Giebel B, Kordelas L, Börger V. Clinical potential of mesenchymal stem/stromal cell-derived extracellular vesicles. Stem Cell Investig 2017;4:84. medicinal products. EMA/CAT/600280/2010 rev.1. 2015.

84. on advanced therapy medicinal products and amending Directive 2001/83/EC and Regulation (EC) No 726/2004.

85. Lener T, Gimona M, Aigner L, et al. Applying extracellular vesicles based therapeutics in clinical trials an ISEV position paper. J Extracell Vesicles 2015;4:30087.

86. Reiner AT, Witwer KW, van Balkom BWM, et al. Concise review: developing best-practice models for the therapeutic use of extracellular vesicles. Stem Cells Transl Med 2017;6:1730-9.

87. Dlouhy BJ, Awe O, Rao RC, et al. Autograft-derived spinal cord mass following olfactory mucosal cell transplantation in a spinal cord injury patient: Case report. J Neurosurg Spine 2014;21:618-22.

88. Théry C, Amigorena S, Raposo G, et al. Isolation and characterization of exosomes from cell culture supernatants and biological fluids. Curr Protoc Cell Biol 2006; Chapter 3:Unit 3.22.

89. Vishnubhatla I, Corteling R, Stevanato L, et al. The development of stem cell-derived exosomes as a cell-free regenerative medicine. J Circ Biomark 2014.

90. Heinemann ML, Ilmer M, Silva LP, et al. Benchtop isolation and characterization of functional exosomes by sequential filtration. J Chromatogr A 2014;1371:125-35. 